\title{
El Conocimiento, Propulsor de los Ciclos Largos de Kondratieff y sus Efectos en la Configuración Mundial
}

\author{
Vega-González L. R', Vega-Salinas. R M.
}

\begin{abstract}
El objetivo de este trabajo es desarrollar un medio sencillo para visualizar la evolución del sistema mundial, a partir del avance del conocimiento. Para lo cual, planteamos que el conocimiento ha sido el precursor de la tecnología y por lo tanto de la evolución social y de la conformación del sistema mundial a lo largo de la mayor parte de la historia. La metodología empleada fue la revisión de los grandes fenómenos globales representativos de la situación de la política internacional, tomando como eje la teoría de ciclos largos de Kondratieff, y siguiendo el planteamiento de Modelski \& Thompson (1996) de la aparente existencia de al menos veinte ondas Kondratieff (K-I a K-20) rastreables. Usando estos elementos teóricos en el contexto global, en conjunto con un modelo propuesto para las relaciones causales entre las variables más representativas del fenómeno, obtuvimos una primera versión de gráficos condensados que permiten visualizar la evolución del sistema mundial fácilmente.
\end{abstract}

Keywords: ciclos kondratieff, sistema mundial, conocimiento.

This work objective is to develop a simple media to visualize the world system evolution based in the knowledge advance. To achieve this, we pose that knowledge has been precursor of technology and therefore of the social evolution and the conformation of the world system during the major part of history. The employed methodology was the revision of the great global representative phenomena of the international politics through the use of the Kondratieff theory of long cycles and following the Modelski \& Thompson (1996) proposal of the existence of at least twenty. Kondratieff waves (K-I to K20) traceable. Using these theoretical elements in the global context, in conjunction with a model proposed for the causal relationships of the most representative variables of the phenomena, we obtained condensed graphics that allow the easy visualization of the evolution of the world system.

Keywords: kondratieff cycles, world system, knowledge.

'Centro de Ciencias Aplicadas y Desarrollo Tecnológico, UNAM, Circuito Exterior S/N, Ciudad Universitaria,A.P. 70-I86, Delegación Coyoacán 045I0, México D.F. México. Coordinación de Vinculación y Gestión Tecnológica. E-mail: Irvg@servidor.unam.mx;

${ }^{2}$ Facultad de Ciencias Políticas y Sociales de la Universidad Nacional Autónoma de México (UNAM). 


\section{Introducción}

El planteamiento básico de este trabajo es que el conocimiento ha sido el detonador de la dinámica de las diferentes variables que conforman el sistema mundial y por lo tanto es el precursor de la evolución y de la innovación social. En esta dinámica de progreso existen muchos reacomodos en el orden y el liderazgo económico en los países que conforman el sistema mundial. El conocimiento es intangible, por lo que una posibilidad para visualizarlo, es a través del estudio de las grandes épocas de evolución tecnológica que han existido a lo largo de la historia del hombre y que han propiciado su bienestar y progreso.

Desde que el hombre apareció en el mundo, aprendió a subsistir y poco a poco convirtió su aprendizaje en conocimiento, el cual era transmitido de generación en generación en las civilizaciones antiguas. El conjunto de saberes de las sociedades más primitivas permitía a los hombres conocer, entender y explicar las cosas y los fenómenos que les rodeaban, obtener sus alimentos, guarnecerse del clima, vestirse y defenderse de otros clanes y de los animales salvajes. Éste tipo de conocimiento práctico o empírico existe desde el principio de la historia humana, es acumulativo, se encuentra en movimiento y cambia constantemente. A este tipo de conocimiento se le conoce como conocimiento técnico. Con el paso del tiempo y con el conocimiento adquirido como herramienta principal, los individuos han formado sociedades y países delimitados geográfica y culturalmente.

Cuando el hombre dejo de ser nómada y se asentó en los poblados que a la postre se convirtieron en ciudades, poseía el dominio de diversas técnicas que al combinarse poco a poco se convirtieron en tecnologías como las de crianza de animales y de la agricultura, a las que posteriormente se les llamó agropecuarias. Después, desarrolló las técnicas y procedimientos para la explotación de la lana y la confección de ropa. Más tarde, con el dominio de la energía y del conjunto de conocimientos acumulados y disponibles, el hombre desarrolló tecnologías más complejas para la producción de cobre, bronce y hierro desde aproximadamente el año 4000 ADC. (Edad de los metales, S/D) hacia el siglo XII AC.

Estas tecnologías poco a poco se difundieron en el mundo permitiendo el desarrollo de nuevas herramientas usadas para la construcción de viviendas, caminos y el almacenamiento de agua, pero también con fines bélicos. Así, pasaron centurias, en las cuales a la par del incremento de la población mundial, se dieron nuevos conocimientos que facilitaron la transportación marítima de mercancías y la conservación de alimentos, desarrollándose cada vez más el comercio internacional. Con el dominio de la navegación en los mares, nuevos grupos sociales tomaron el liderazgo mundial. Un buen ejemplo son los fenicios quienes durante los siglos 1200-539 AC dominaron el comercio marítimo de vinos, tintes púrpura para textiles y metales al oriente del mar mediterráneo.

Por supuesto que además de los conocimientos técnicos y tecnológicos prácticamente todos los pueblos de la humanidad desarrollaron conocimiento científico.Así, sabemos que las antiguas civilizaciones de Mesopotamia, los egipcios, los fenicios y los griegos, tenían diversos conocimientos de astronomía, geometría, geografía, física, química y medicina.

Hacia el año 753 AC los romanos se convirtieron en la primera potencia mundial. En Roma se desarrollaron conocimientos científicos en astronomía, medicina y salud y muchos conocimientos tecnológicos tales como el dominio de tecnologías para fabricar tubos de plomo y cemento y técnicas como la cerrajería, lo que les permitió desarrollar sus egregias edificaciones. Del otro lado del mundo, en América, la civilización Maya (I000 AC- 187 DC), en sus periodos preclásico, clásico y posclásico, desarrolló extraordinarios conocimientos en astronomía y matemáticas.

Durante muchas centurias de nuestra era, en el mundo se vivió un periodo de oscurantismo, en términos del avance del conocimiento debido a la marcada influencia de la religión en la ciencia, Besant (1898, pag.3),

Lo anterior propició que durante muchos años los avances en el conocimiento se dieran muy lentamente. En Europa no fue sino hasta que a partir de los postulados de Descartes y de Bacon, (Bello, 2003; Farrington, 2005) durante el renacimiento se sentaron las bases del método científico en el que se propone que el conocimiento, se construye a través de la racionalidad, planteando preguntas de investigación mediante las cuales se crean hipótesis que al comprobarse sucesiva y permanentemente se convierten en teorías. En la edad moderna al conocimiento construido a partir de la aplicación del método científico se le conoció como conocimiento científico. Es interesante notar que aún cuando las hipótesis sean refutadas y resulten ser falsas, estos resultados se convierten en conocimiento valioso. Haciendo uso intensivo del método científico, a lo largo de las últimas centurias del siglo pasado, el hombre fue generando conocimiento básico y fundamental, con lo cual hemos llegado hasta nuestros días.

En la Figura I, pueden verse los tres tipos de conocimiento al que llamaremos de supervivencia y progreso (S\&P) desarrollados por el hombre a lo largo de la historia. Dentro del conocimiento de S\&P no incluimos ni el obtenido de estudios filosóficos, ni el derivado de las humanidades, ni el religioso. 
En resumen, podemos decir que tanto el conocimiento técnico como el conocimiento científico han existido a lo largo de toda la historia y a partir de la interrelación de ambos se ha desarrollado la tecnología, que a su vez ha servido de soporte para la generación de nuevas técnicas y conocimiento científico en un camino de cambio y mejora incesante.

\section{Relaciones causales entre el conocimiento y la configuración mundial}

De acuerdo con Berger \& Luckmann, (2006), el conocimiento a lo largo de la historia ha tomado un papel activo en la construcción de la realidad social, basados en este planteamiento, en este trabajo proponemos que existen relaciones causales entre el conocimiento, la ciencia, la tecnología y la economía, las cuales se dan a través de la innovación continua y la emergencia de nuevos productos. La innovación se alcanza con la difusión de los nuevos productos en el mercado. Su aceptación, adaptación y asimilación en los distintos sectores de la sociedad, tiene efectos que con el tiempo suficiente provoca cambios sustanciales, ajustes y nuevas tendencias la misma.

A lo largo de los años, los distintos países que conforman el mapa internacional han establecido relaciones de intercambio comercial, cultural, y social entre sus individuos y organizaciones. La ciencia política estudia la evolución, el desarrollo y la situación que guardan las relaciones entre los diferentes actores de la estructura internacional. Actualmente, los cambios en la conformación hegemónica mundial son constantes debido a que el mundo se encuentra inmerso del complejo fenómeno de la globalización. Entonces, es previsible que existan relaciones causales entre la conformación del sistema mundial (geopolítica) y el conocimiento humano.

En la Figura 2 se presenta un modelo simplificado de la forma en la que se dan las secuencias de causa-efecto de la actividad humana en el macro sistema mundial. El conocimiento es el punto de partida y el punto de cierre, por lo tanto, la generación continua del mismo permite que el sistema funcione como un sistema cerrado con una dinámica continua. Como dijimos en el apartado anterior, entendemos por conocimiento al conjunto de saberes que ha desarrollado y usado el hombre para sobrevivir y progresar a lo largo de su historia y que específicamente se refiere a las distintas técnicas, procesos, procedimientos, dispositivos, aparatos y sistemas para conservar o producir tanto alimentos como elementos diversos necesarios para llevar a cabo sus actividades en la vida diaria.

Al conjunto de conocimientos desarrollados por el hombre para lograr su bienestar a través de la producción de sus satisfactores, tales como: su casa, vestido, alimentos, medicamentos, entretenimiento; entre muchos otros, comúnmente se le conoce como plataforma tecnológica.

Por lo tanto, la plataforma tecnológica, al igual que el conocimiento es cambiante, creciente, $y$ aditiva; es decir, requiere de las plataformas previas para construir las actuales, y por su uso define permanentemente nuevos sistemas de producción ya sea artesanal, semi-automáticos o automáticos que

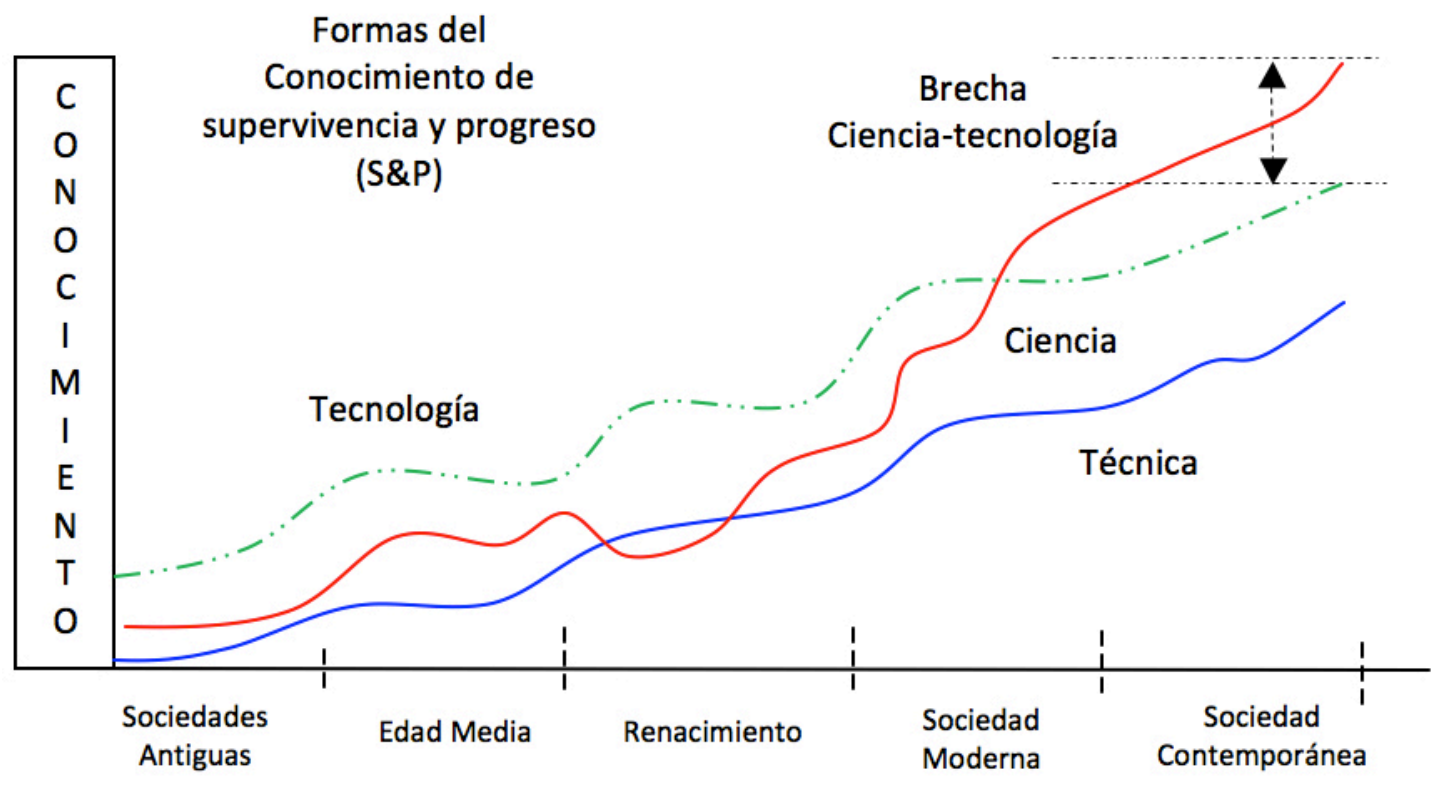

Figura I. Relaciones entre el conocimiento técnico, científico y tecnológico

ISSN: 07I 8-2724. (http://www.jotmi.org)

Journal of Technology Management \& Innovation (c) Universidad Alberto Hurtado, Facultad de Economía y Negocios. 
son adoptadas por nuevas empresas e industrias en las que se procesan los recursos naturales y se producen nuevos productos, en la forma de bienes y servicios, los cuales con su difusión dinamizan la economía dando pie a nuevas actividades comerciales e industriales.

Las plataformas tecnológicas usadas en los nuevos procesos productivos en conjunto con los productos tecnológicos de consumo personal o familiar, definen las tendencias tecnológicas que a su vez impulsan estrechamente las tendencias económicas mundiales.

La interacción de todas estas macro-variables provoca cambios sociales a nivel de cada uno de los países, de sus regiones geográficas y finalmente llega a provocar reacomodos y cambios en la configuración que guardan los distintos países del orbe.

Las reconfiguraciones nacionales, regionales, internacionales que se dan entre los países, propician el cambio constante de las formas y del número de interacciones que se dan entre los países que constituyen el sistema mundial. Entonces, el campo de acción de las relaciones internacionales es el estudio de la adaptación y el ajuste dinámico de las interacciones humanas, comerciales, políticas, diplomáticas, de alianzas tecnológicas, culturales; etcétera, que se dan entre los diferentes países del orbe.

\section{La teoría de las ondas largas de Kondratieff}

En la Figura 2 puede verse que en el corazón de los procesos de cambio que se dan entre el conocimiento y la conformación mundial se encuentran los intensos cambios de las variables tecnológicas y las económicas. Los cambios en las tendencias de la economía mundial impulsadas por los cambios en las tecnologías innovadoras que han dado lugar a las nuevas plataformas tecnológicas, fueron estudiadas por la teoría de las ondas largas olas o ciclos largos de desarrollo económico de Nikolái Dmítrievich Kondratieff. (Gottfried, 1956). Esta teoría, basada en la observación de los ciclos que se dan en todos los órdenes de la naturaleza, y en el reconocimiento de que el progreso humano es una la tendencia irreversible, es muy útil para entender los enormes procesos que suceden y que han sucedido en el mundo. El tema central de esta teoría, es la relación entre el desarrollo social y las innovaciones tecnológicas; de manera que la teoría de los ciclos largos (LC, por sus siglas en inglés), es precursora de los estudios sobre el papel social de la tecnología.

El principal trabajo de Kondratieff sobre las ondas largas fue realizado en 1920, (Kondratieff, 1926), entre sus resultados resalta que aunque existen ondas económicas de distintas frecuencias, las ondas envolventes principales tienen ciclos recurrentes de cerca de cincuenta años en los que el crecimiento económico se explica a partir de la difusión de sucesivas revoluciones tecnológicas, cada una de las cuales rep-

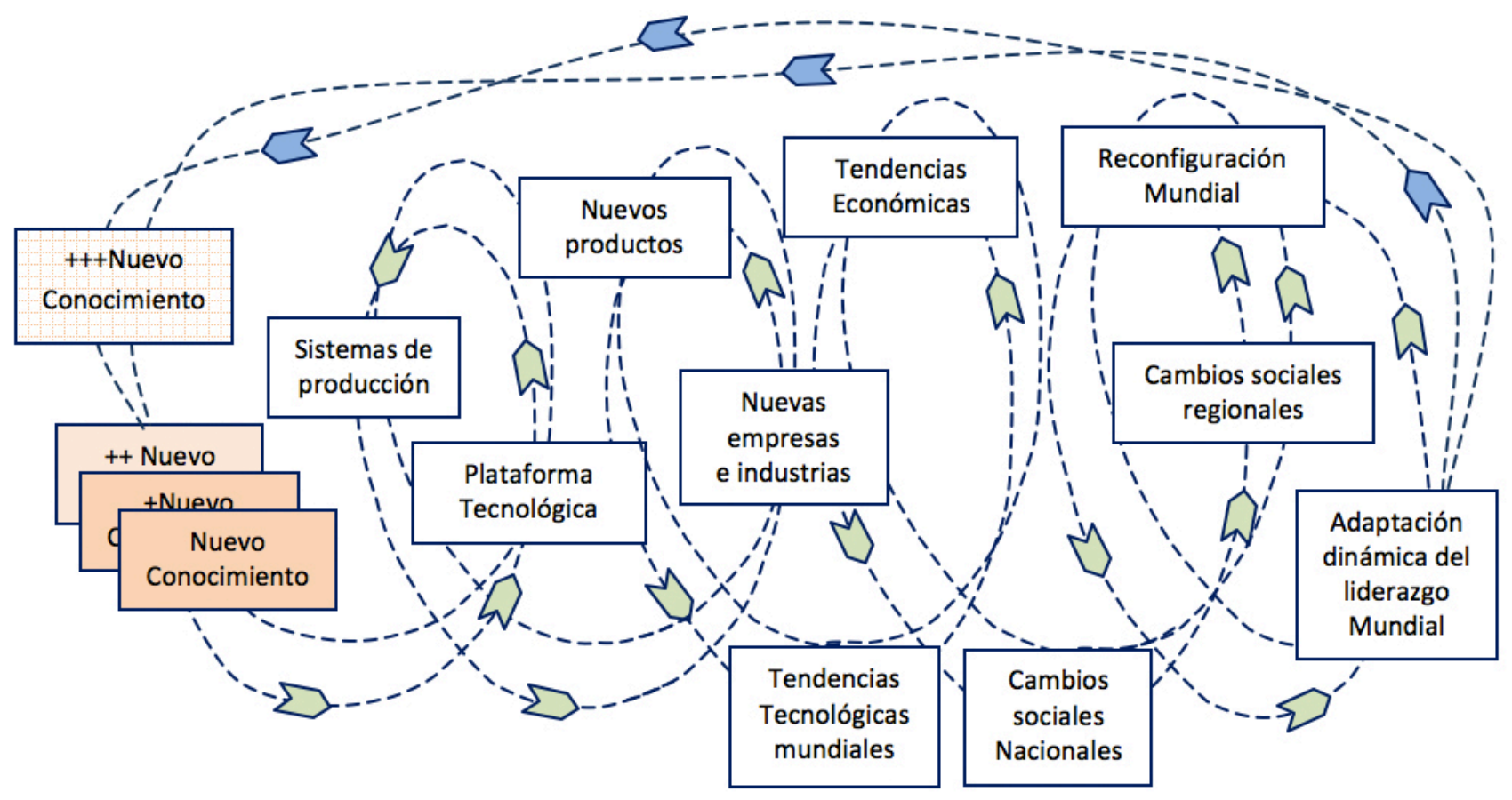

Figura 2. Relaciones causa-efecto dinámicas en el sistema mundial cerrado

ISSN: 07 I8-2724. (http://www.jotmi.org)

Journal of Technology Management \& Innovation @ Universidad Alberto Hurtado, Facultad de Economía y Negocios. 
resenta incrementos muy importantes en la productividad de prácticamente todos los sectores del aparato económico. Posiblemente la causa de la forma cíclica del proceso es que después del impulso inicial para la economía provocado por el conjunto de innovaciones tecnológicas de la revolución tecnológica en curso, la economía mundial alcanza en unas décadas una nueva cima mientras que poco a poco se reestructura el marco socio-institucional, tanto en el plano nacional como en el internacional. Una vez que la onda de impulso económico se estabiliza y la sociedad se adapta al uso permanente de las nuevas tecnologías, nuevamente decrece el movimiento económico hasta que se alcanza el valle de la onda. Sin embargo, durante todos estos años, tanto las empresas establecidas como muchas nuevas, $y$ en el caso de la sociedad contemporánea, diversas instituciones de investigación y desarrollo a nivel mundial continúan su trabajo generando otras tecnologías en la búsqueda del progreso y el bienestar humano. Al ser lanzados al mercado los nuevos productos tecnológicos dan inicio a un nuevo ciclo económico. Notablemente las transformaciones sociales e institucionales que acompañan al proceso económico determinan el "modo de crecimiento" de la siguiente onda larga. Entonces, una onda Kondratieff se puede definir, como el auge y caída de un modo de crecimiento y crisis tecnológica así como la ardua transición de un modo de crecimiento económico al otro. Según Modelski (1987), las ondas-K son atributos de la economía mundial que se hacen más visibles en los datos de la producción internacional que en las economías domésticas.

El fenómeno identificado por Kondratieff es de una gran relevancia, ya que su estudio podría ayudar a explicar la fenomenología del sistema mundial si fuese consistente a lo largo de la historia y pudiera convertirse en teoría. Interesados en este problema y ante la falta de herramientas teóricas que coadyuven a explicar la fenomenología del sistema mundial, Gunder, et al, (1993);Wallerstein (1984, 20 I la, 20l lb). Modelski (199I), Amin (20II) y Amin et, al. (2006), se preguntaron ihasta qué época de la historia del sistema mundial es posible detectar las oscilaciones tecnológico-económicas identificadas por Kondratieff?, y si ¿Es O no posible identificar las principales características sistémicas del sistema mundial moderno antes de 1500? En muchos de los casos se argumenta que nuestro sistema mundial tiene diferencias específicas desde el año 1500 y hasta nuestros días. En un estudio posterior, Modelski \&Thompson (2008) identificaron que desde el año $870 \mathrm{DC}$ hasta la fecha han sucedido 19 ondas Kondratieff (KI-KI9) y nueve ciclos largos (LCILC9). Bajo este concepto, estamos viviendo la onda $\mathrm{K} 20$ y la segunda parte del LCI0. Existen diversas posiciones, por ejemplo, interesado en el futuro del sistema mundial, Nefiodow (20I3) indica que solo se han vivido cinco ciclos largos (LC) con características perfectamente identificadas desde el año $I 780$ y en breve iniciaremos el sexto ciclo largo.
En este trabajo adoptaremos la propuesta de Modelski \& Thompson (Op. Cit.) de que la fenomenología de las ondas $\mathrm{K}$ y los ciclos largos (LC) puede extenderse hasta finales del primer milenio de nuestra era y haciendo uso de las relaciones cauda-efecto del sistema mundial consideradas en la Figura 2, a continuación trataremos de explicar gráficamente la forma en la que el conocimiento, ha sido el propulsor de los ciclos largos de Kondratieff y cuáles han sido sus efectos en la configuración mundial.

\section{I Periodo de los años 870- I250 DC}

En la Figura 3, elaborada a partir de Modelski \& Thompson, (1996, pag. 137; Tabla 8.5), se presenta el periodo de los años 870- 1250 DC, correspondiente a las ondas Kondratieff $\mathrm{K}-\mathrm{I}$ a K-6 que formaron los ciclos largos LCI a LC3. Los años 870 al I I 20 DC corresponden a las ondas K-I a K-4 y/o a los ciclos largos LCl y LC2, caracterizados por el oscurantismo de la Edad Media durante los cuales fueron muy pocos los conocimientos científicos en Europa, y los pocos existentes se dieron sobre todo en los países del medio y lejano oriente. En esa época se desarrollaron dispositivos tecnológicos como la brújula magnética y los relojes que contribuyeron a mejorar la navegación de los mares.También se desarrollaron molinos hidráulicos y papel moneda impreso. En el mundo existían principalmente monarquías feudales y se daban enfrentamientos e invasiones de los países bárbaros. La economía era agrícola y el comercio se realizaba principalmente por tierra. Según Modelski \& Thompson (Op. Cit.), el liderazgo mundial lo ostentó la Dinastía Song del Norte de China pasando posteriormente a la Dinastía Song del Sur de China al fin del primer milenio y en el inicio del nuevo. (Needham \& Wang, 2004) En esta nueva época se desarrollo la construcción naval con lo que se propició el establecimiento de nuevas rutas marítimas. Entre el año II 20 al I250 DC el comercio marítimo tomo un gran esplendor y a través de la construcción de flotas de navíos y las Ferias Comerciales de Champagne, Génova Italia alcanzó el liderazgo económico mundial y con su mejora económica pudo repeler con éxito los ataques de los musulmanes. En ese tiempo se desarrollaron el compás de navegación magnético y el vidrio plano y los tintes que caracterizarían tanto el arte en vitrales como los textiles finos europeos. Durante el primer cuarto del segundo milenio se libraron en Europa las nueve (9) guerras santas de los estados cristianos europeos contra los sarracenos, conocidas como las Cruzadas. A principios del siglo XII con el establecimiento de las primeras universidades y la introducción del sistema de numeración indo-arábigo en Europa por Leonardo de Pisa (Fibonacci), dio inicio la edad media y el renacimiento. 


\subsection{Periodo de los años 870- I 250 DC}

En la Figura 4, elaborada a partir de Modelski \& Thompson, (Op. Cit.), se presenta el periodo que va de los años 1250 al I580 DC correspondiente a las ondas Kondratieff K-7 a K-I 2 que formaron los ciclos largos LC4 a LC6. Durante todo este periodo, la economía mundial se caracterizó por la expansión marítima comercial. Las ondas K-7 a K-9 cubrieron el renacimiento de la edad media iniciado a principios del milenio y extendido hasta mediados del siglo. (LC4 y primera parte de LC5). A excepción de los años 1337 al 1453, durante los cuales se dio un gran vacío en la ciencia debido a la peste negra y la guerra de los cien años, hubo un intenso crecimiento de las ciencias matemáticas, óptica, astronomía, medicina, física, alquimia. En el segundo cuarto del segundo milenio se invento la exclusa que contribuyo a mejorar la navegación de los mares con diferentes niveles y se desarrollaron en Venecia las conocidas flotas de galeones que surcarían todos los mares conocidos, con la idea de controlar el comercio de especias de oriente, estableciendo una relación comercial muy compleja con los Turcos. En el viejo mundo las monarquías feudales se convirtieron en monarquías autoritarias.
Con sus flotas navales y el desarrollo de los cañones y la artillería pesada en Europa, Portugal asumió el liderazgo mundial durante los años 1350 a 1492 DC. Estos años también se caracterizaron por el control del comercio de especies y el inicio de las expediciones de coloniaje para capturar el oro de Nueva Guinea. Con la toma de Constantinopla en 1453 por los turcos otomanos se da una transición de las monarquías autoritarias hacia las naciones estado. En la misma época se estableció el sistema veneciano de patentes. El descubrimiento de América por el genovés Cristóbal Colón en 1492 dio inicio al ciclo largo LC-6 formado por las ondas K-II y K-12. Este periodo se caracterizó por la fiebre de colonización de los países europeos en América, Asia, África y Oceanía. El objetivo del colonizador fue la explotación de los recursos naturales y la mano de obra de sus colonias.

Con el tremendo impacto a nivel mundial propiciado por el desarrollo de la imprenta de tipos móviles por el alemán Johannes Gutemberg hacia 1450 y su localización geográfica estratégica, en el centro de Europa, Alemania tomo el liderazgo económico mundial debido a su comercio con los países circundantes al mar Báltico, en el norte de Europa y con Asia. (Modelski \& Thompson, Op. Cit.).

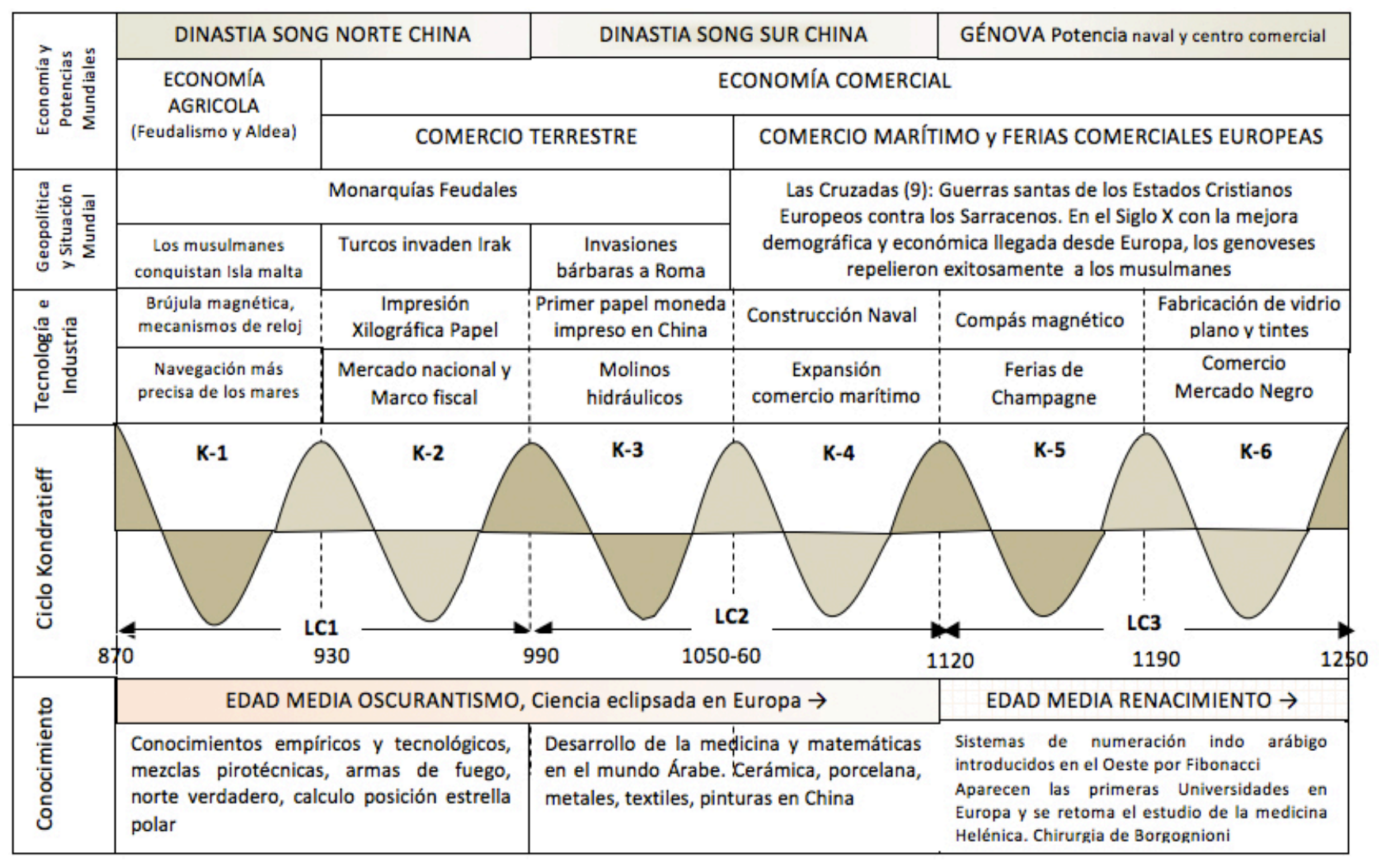

Figura 3. Conocimiento, ciclos largos (LCI-LC3), y su relación con la tecnología, la geopolítica y la economía.

ISSN: 07 I8-2724. (http://www.jotmi.org)

Journal of Technology Management \& Innovation (c) Universidad Alberto Hurtado, Facultad de Economía y Negocios. 
A partir de 1500 y hasta 1580 DC; es decir, durante el siglo $\mathrm{XVI}$ se gestó la Revolución Científica de la Edad Moderna, caracterizada por un desarrollo científico impresionante.Algunos exponentes del desarrollo científico de esta época fueron Miguel de Servet con sus Sistema Circulatorio Pulmonar, Nicolás Copérnico con su modelo heliocéntrico del Universo, Paracelso, alquimista, medico y astrologo Suizo que reclamó la transmutación del plomo en oro y el notable polímata del renacimiento italiano, así como Leonardo $\mathrm{Da}$ Vinci, quien fue uno de los mejores pintores de todos los tiempos y genio universal, considerado como la persona con el mayor número de talentos en múltiples disciplinas que jamás ha existido.

\subsection{Periodo de los años I580- I9|4 DC}

La Figura 4, que fue también elaborada a tomando como referencia los ciclos identificados por Modelski \& Thompson, (Op. Cit.), presenta el periodo que va de los años 1580 al I9I4 DC correspondiente a las ondas Kondratieff $\mathrm{K}-13$ a $\mathrm{K}-\mathrm{I} 8$ formando los ciclos largos LC7 a LC9.

Durante los siglos XVI y XVII; ondas K-I3 y KI4 (LC7), la Gran Bretaña adquirió y mantuvo el liderazgo económico y político internacional debido a su claro dominio marítimo comercial así como por su dominio de los procesos de beneficio de minerales. Su dominio a nivel mundial quedo de manifiesto con el establecimiento de trece colonias de migrantes ingleses en Norteamérica, así como colonias en la India, África Sudamérica y Australia. En esa época se libraron las guerras anglo-española y la guerra de los tres reinos entre Escocia, Irlanda e Inglaterra. Como parte de la edad moderna, en el siglo XVI se dio un avance espectacular en la Física clásica con las fundamentales aportaciones de Descartes y Bacón para establecer el método científico, el establecimiento de las bases de la mecánica clásica y la gravitación universal por Isaac Newton, padre del cálculo integro-diferencial; la invención del telescopio por Galileo, que después sería mejorado por Newton, y los trabajos de Leeuwenhoek precursores de la microbiología y la biología celular.

Impulsada por la primera ola de la revolución industrial con la era del carbón y el vapor (I780 - 1840), la Gran Bretaña también lideró al mundo durante el siglo XVIII. Este fenómeno fue reconocido en los trabajos de Schumpeter (20I2). Al llegar el siglo XIX, y con la segunda revolución industrial, inicialmente llamada revolución técnica (Duijn, 1983), el liderazgo económico mundial fue adquirido por los Estados Unidos de Norteamérica. Este siglo se caracterizó por la edad de los ferrocarriles y la producción en serie (1840 1890), el dominio de los procesos de fundición de acero, la

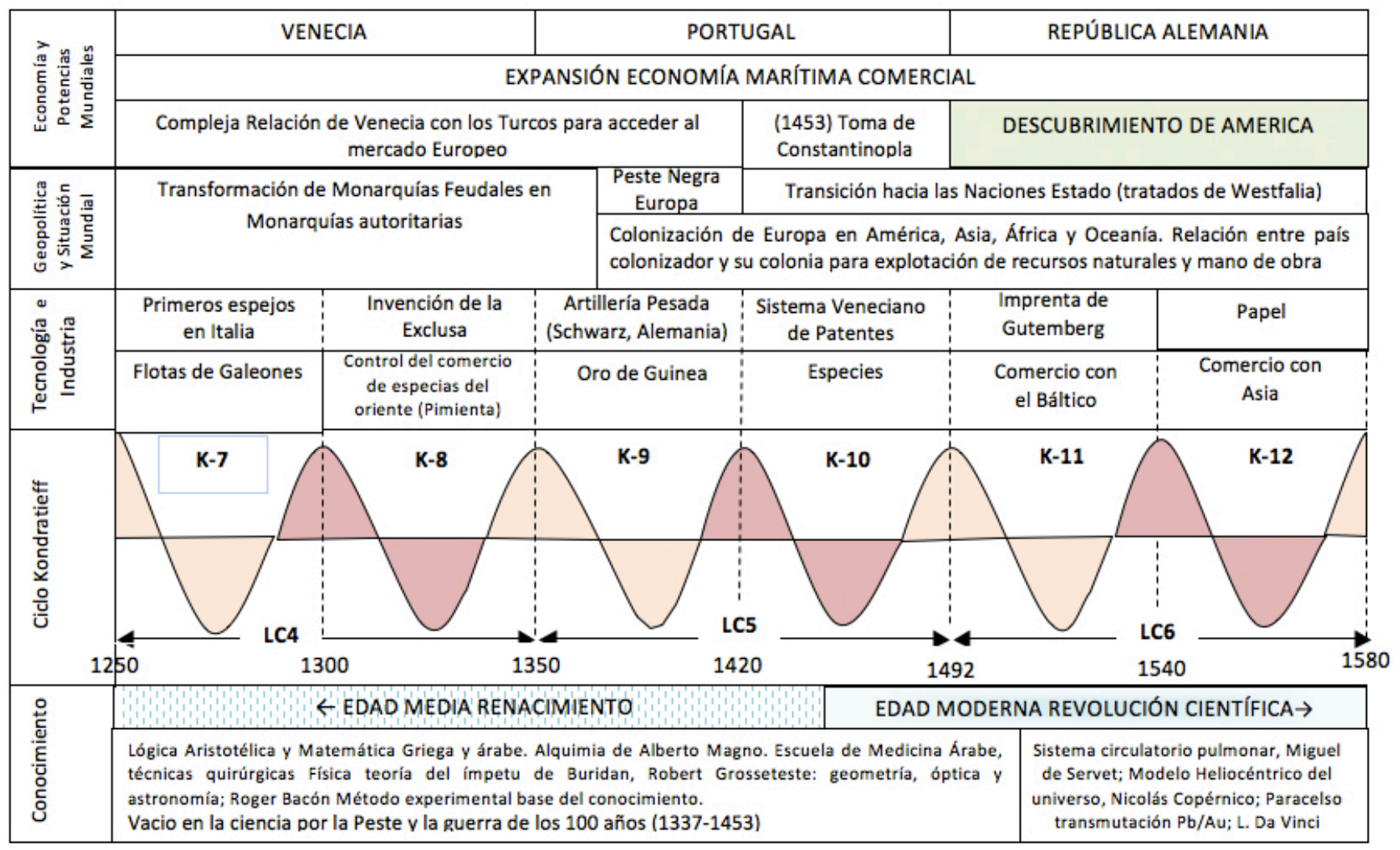

Figura 4. Conocimiento, ciclos largos (LC4-LC6), y su relación con la tecnología, la geopolítica y la economía.

ISSN: 07I 8-2724. (http://www.jotmi.org)

Journal of Technology Management \& Innovation (c) Universidad Alberto Hurtado, Facultad de Economía y Negocios. 
refinación de petróleo, y el cemento Portland; y ya entrado el siglo XX con la era de la electricidad (1890 - 1940). Con el reconocimiento mundial del Estado Moderno y el derecho a la propiedad privada absoluta, la economía mundial se caracterizó por el desarrollo del capitalismo en el cual se consideró al trabajo como el factor de la producción principal, por encima de la disposición de los recursos naturales. En I8I5, con la derrota de Napoleón en Waterloo y con la revolución Francesa se requirió de la restructuración de toda Europa. Durante el Siglo XIX se presentaron las guerras de independencia de los países hispanoamericanos Venezuela, Ecuador, Panamá, Bolivia, Chile, México y los países centroamericanos. A finales del siglo XVIII como preámbulo a la edad contemporánea se presento en algunos países de Europa el siglo de las luces, el cual fue denominado así por su declarada finalidad de disipar las tinieblas de la humanidad mediante las luces de la razón. A principios del siglo $X X$, ya dentro de la edad contemporánea el mundo recibió las aportaciones al conocimiento científico de Charles Darwin con su teoría de la evolución, la teoría atómica de Dalton, y la Psicoanalítica de Sigmund Freud, entre muchas otras.

\subsection{Periodo Años 19|4- 2026 DC}

En la Figura 6, elaborada tomando como base las referencias de Nefiodow (2006), se muestra el periodo de la edad contemporánea concerniente a las ondas $\mathrm{K}-19$ y a la K-20, actualmente en curso, ambas conformando el ciclo largo LCl0, cuyo máximo se estima hacia el año 2030.

Los Estados Unidos de Norteamérica han mantenido el dominio económico durante todo el siglo $X X$ y lo que va del siglo XXI, reconocido plenamente como la primera potencia mundial. El sistema económico prevaleciente en ambos siglos ha sido el capitalismo imperial y posteriormente neoliberal caracterizado por el gran consumo en masa y la movilidad individual. Las dos grandes guerras mundiales libradas entre 1914 y 1945, la primera fase de la economía industrial, como economía de la guerra, se continuó con el desarrollo de los procesos de transformación de la petroquímica secundaria y los de manufactura de la industria metal mecánica. En la primera mitad del siglo $X X$ se desarrolló la industria automotriz, el radio y la televisión basados en bulbos o válvulas al vacío, y el radar como producto de los desarrollos de la guerra. La era espacial comenzó a mediados de siglo dando paso a la carrera ruso-norteamericana para poner las redes de satélites artificiales en órbita. El aspecto más distintivo del conocimiento aportado por los científicos en la edad contemporánea fue el desarrollo de la Física Moderna. El crecimiento del conocimiento científico se incrementó sustancialmente, algunas aportaciones relevantes de la primera mitad del siglo XX fueron la teoría de la relatividad de Einstein, el dominio de la energía atómica y las centrales nucleoeléctricas, los antibióticos de Fleming, la determinación de la función biológica del ADN y la industria aeroespacial. Para la segunda mitad del siglo se llevó a cabo el proyecto del genoma humano, las redes satelitales y de telecomunicaciones, los aceleradores de partículas, las supercomputadoras y las computadoras cuánticas. Durante la segunda mitad del siglo $X X$, en plena guerra fría, con el desarrollo pleno de las industrias farmacéutica, agropecuaria y biotecnológica, se dio la segunda fase de la economía industrial en la cual se dio un desarrollo intensivo de dispositivos tecnológicos electrónicos, los transistores, los amplificadores operacionales, los microprocesadores los sistemas láser. La computadora personal (PC) fue lanzada al mercado a nivel mundial durante los 80 's del siglo pasado y durante los 90 's la red mundial de redes (WWV, por sus siglas en inglés) o Internet y los teléfonos celulares. Esto impulsó la nueva revolución tecnológica de la información y las comunicaciones (TIC's). (Freeman, 1985). El mundo se torno en una aldea global en la que la comunicación entre personas distantes localizadas en cualquier parte del mundo podía darse instantáneamente. (Mc. Luhan, 1994). Es muy claro que las TIC's ofrecieron una nueva infraestructura tecnológica mediante la cual cambiaron las condiciones para la producción y la distribución del conocimiento. Respecto a la situación mundial, hacia finales del siglo $X X$ se dio la caída del muro de Berlín, el fin de la URSS y la guerra del golfo pérsico.

Actualmente, a principios de la segunda década del siglo XXI, como nunca antes se generan enormes cantidades de datos y de información la cual se analiza y procesa y se combina para generar nuevo conocimiento; además, haciendo uso de las redes de telecomunicaciones, la información está disponible instantáneamente en cualquier sitio y en cualquier lugar, por lo que sin lugar a dudas nos encontramos plenamente en la sociedad y la economía del conocimiento.

En la nueva sociedad el conocimiento es el principal factor de la producción y sus componentes principales son la ciencia, la tecnología y la técnica requeridas para su aplicación por una gran diversidad de agentes, instituciones y relaciones. Por ejemplo, los procesos de transformación son parte del sistema de ciencia-tecnología-industria el cual es cada vez más poderoso y más autónomo frente a otros sistemas, tales como el político, el cultural e incluso el económico. (Lamo de Espinoza et al., 1994).

Se espera que hacia el año 2030, en la cresta de la onda K-20 y al final de la LCIO se presente como parte de la economía del conocimiento, la bioeconomía, en la que se espera que se dé el fenómeno de la bioconvergencia y que el mercado de la salud se convertirá en el detonador de la economía mundial. En todos los casos el conocimiento es identificado como el nuevo factor de la producción. Respecto a la situación mundial, desde la última década del siglo $X X$ y durante la primera década del siglo XXI se han firmado 


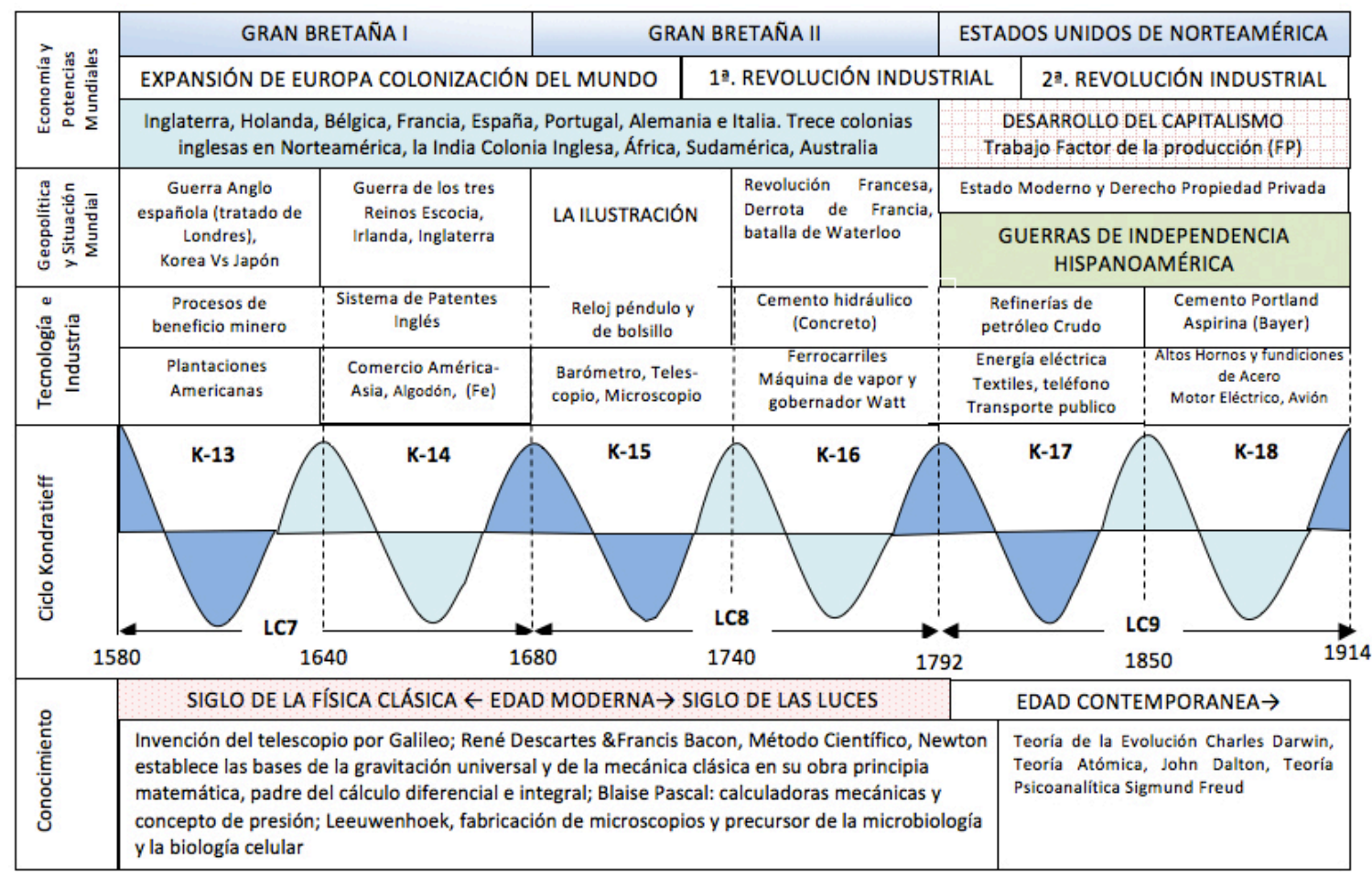

Figura 5. Conocimiento, ciclos largos (LC7-LC9), y su relación con la tecnología, la geopolítica y la economía.

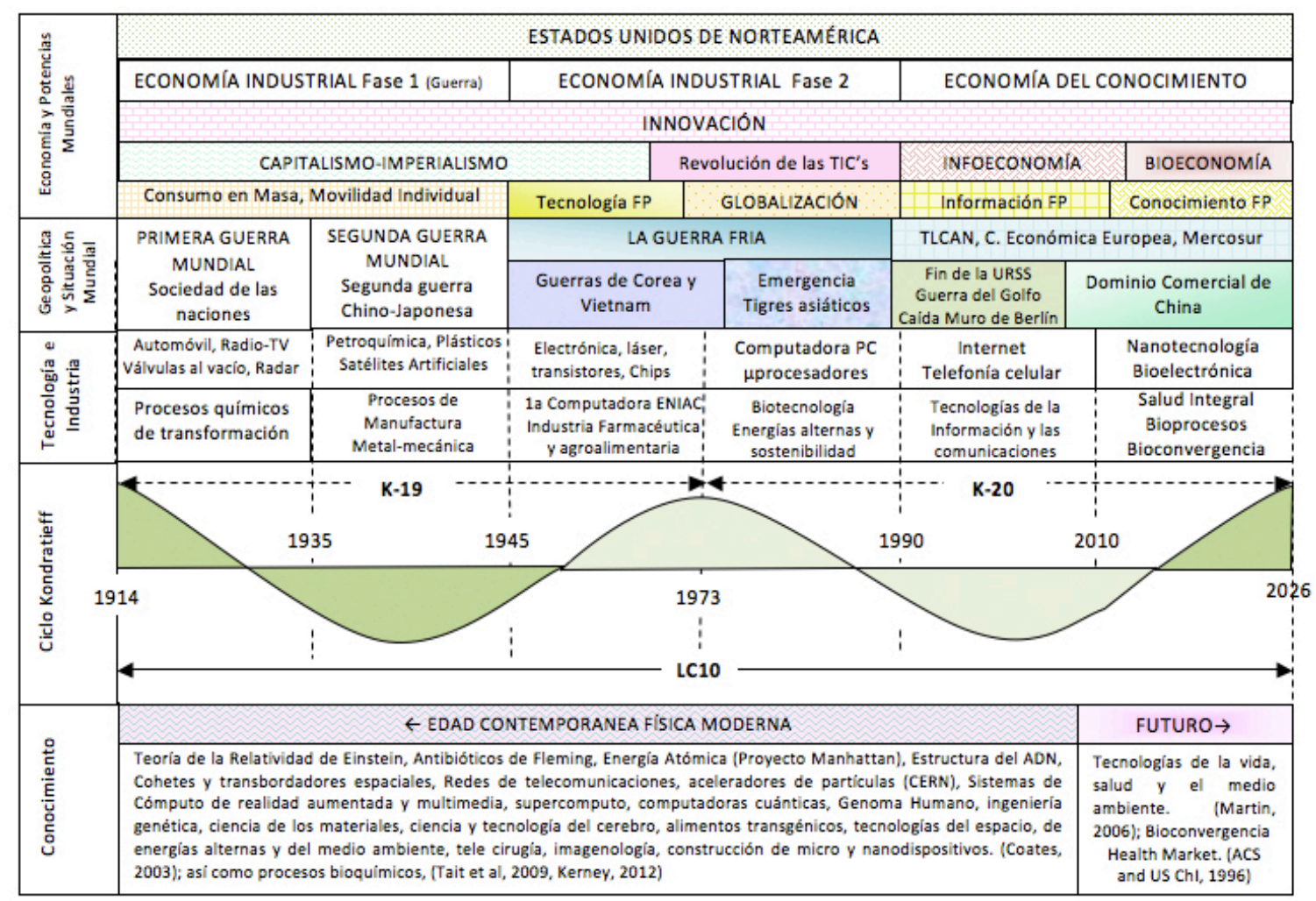

Figura 6. Conocimiento, ciclos largos (LCI0), y su relación con la tecnología, la geopolítica y la economía.

ISSN: 07 I8-2724. (http://www.jotmi.org)

Journal of Technology Management \& Innovation (C) Universidad Alberto Hurtado, Facultad de Economía y Negocios. 
diferentes tratados comerciales como el Tratado de Libre Comercio de América del Norte (TLCAN), y se estableció la Comunidad Económica Europea y el Mercosur. También se ha notado la emergencia del dominio comercial de China, por lo que muchos especulan sobre la posible toma del liderazgo económico mundial por este país hacia el año 2050 dentro de la onda $\mathrm{K}-2 \mathrm{I}$ y el LCII.

En resumen, la sociedad contemporánea se ha caracterizado por el intenso desarrollo de la ciencia y tecnología, las cuales mantienen una brecha permanente lo que impulsa la investigación y desarrollo tecnológico continuos. Tal vez el fenómeno que se ha convertido en la característica principal de los años que van desde finales del siglo $X X$ y a principios del siglo XXI ha sido el intenso cambio tecnológico que ha impulsado el fenómeno de desarrollo y difusión permanente de nuevos productos en el mercado, conocido como innovación.

\section{La Innovación abierta y la nueva situación mundial}

La visión internacional de las relaciones entre los países durante la Guerra fría, desde finales de los años 40's hasta la década de los años 90's del siglo pasado, estaba centrada en el Estado. Posteriormente se dieron intensos cambios estructurales dentro del sistema internacional debido a la proliferación de nuevos actores internacionales más allá del Estado, y al nacimiento de otros entes con capacidad de decisión e influencia dentro del mismo. Hoy en día también se relaciona con la aparición, la consolidación y la interrelación de las organizaciones internacionales, de las empresas transnacionales, de las organizaciones no gubernamentales y también con la movilidad que tienen los mismos individuos. Además podemos decir que los medios de comunicación y las sofisticadas tecnologías modernas de la información han facilitado las relaciones, provocando que las nuevas estructuras internacionales sean extraordinariamente más complejas, dentro del fenómeno de la globalización. La investigación, el desarrollo tecnológico y la innovación (ID+i) se han convertido en temas de interés en las agendas internacionales ya que son propulsores de la economía y por lo tanto de nuevas construcciones sociales.

Desde una perspectiva social la generación de nuevo conocimiento se presenta como el motor del desarrollo y del progreso, por ello es que la investigación y la innovación se encuentran bajo una dinámica de cambio constante.

La característica principal de la sociedad del conocimiento es la forma en la que éste se incorpora en los individuos, las organizaciones y las empresas, o bien en máquinas o artefactos. (Corona \& Jasso, 2005). Indudablemente que en la actualidad para dominar nuevos mercados se requiere del desarrollo de nuevos productos y por lo tanto de nuevo conocimiento. Drucker (2005) menciona, que en la nueva sociedad "el verdadero recurso dominante y factor de producción de riqueza absolutamente decisivo ya no es ni el capital, ni el trabajo, ni los recursos naturales, sino el conocimiento".

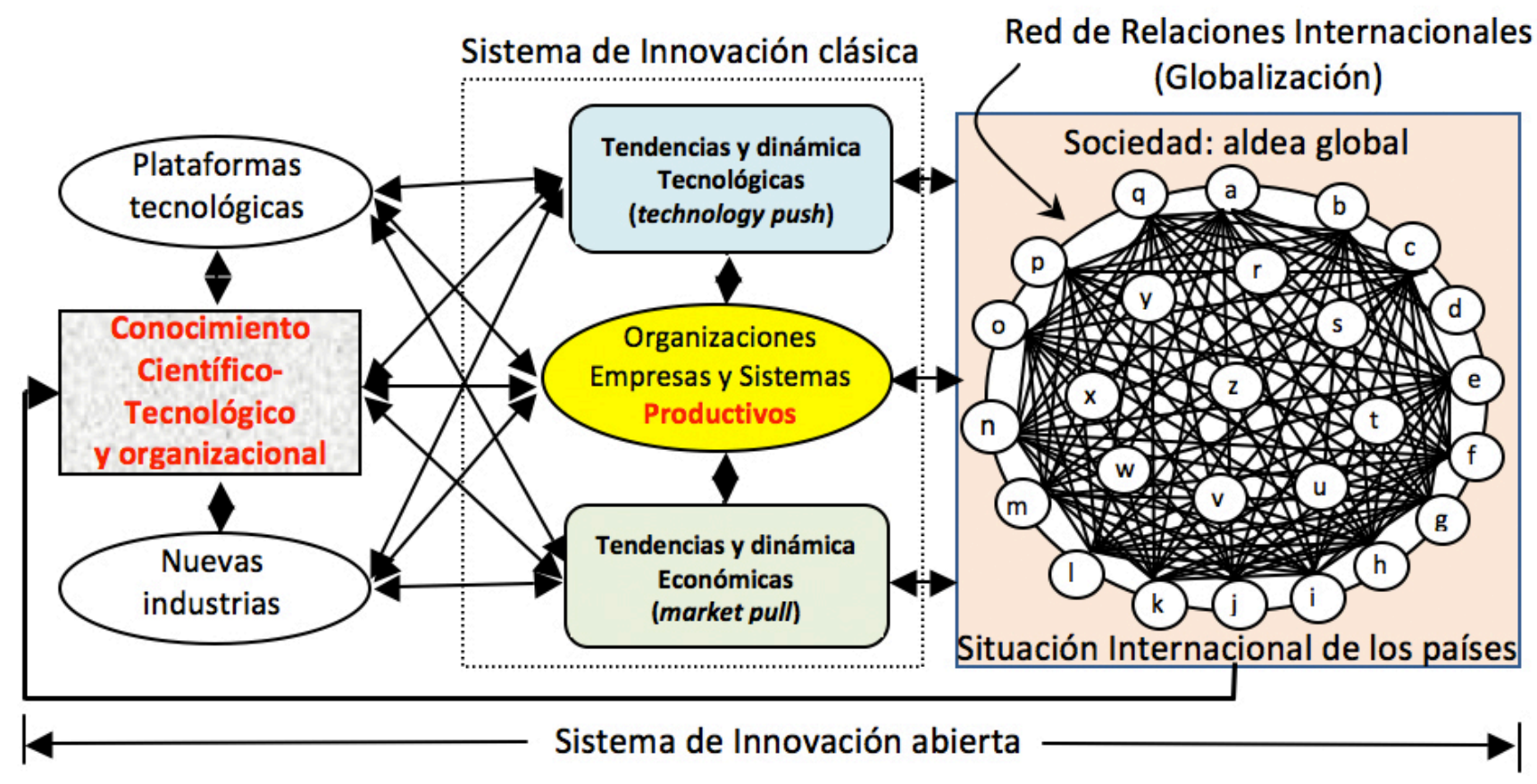

Figura 7. Relaciones entre la innovación abierta y la situación internacional

ISSN: 07I 8-2724. (http://www.jotmi.org)

Journal of Technology Management \& Innovation @ Universidad Alberto Hurtado, Facultad de Economía y Negocios. 
En la figura 7 puede verse que el conocimiento, con un uso productivo, es un elemento clave del funcionamiento económico y por lo tanto, la evolución de nuevas formas de conocimiento tienen como resultado la creación de nuevos actores en el concierto internacional quienes juegan nuevos papeles y en consecuencia se les atribuyen nuevas funciones. Estos cambios de organización implican un giro en la conformación del sistema mundial y en la forma de vida de las sociedades.

La forma en la que se da la innovación en los distintos países que conforman el sistema mundial incide en la forma en que el sistema se reacomoda y en los ajustes y relaciones que se dan entre los distintos países del orbe y las potencias líderes mundiales. En ese sentido, hay que señalar que a lo largo de la primera década del Siglo XXI se ha venido dando un nuevo sistema de innovación abierta caracterizado por el uso intensivo de las tecnologías de la información y las comunicaciones (TIC); tales como las redes de telecomunicaciones, de Internet, la telefonía celular, etc. La génesis de este proceso fue el establecimiento de las alianzas estratégicas entre diferentes organizaciones de una misma comunidad económica y la integración del conocimiento aportado por cada uno de los aliados, usando también sus ventajas competitivas y/o geográficas para crear nuevos productos y servicios que en el momento oportuno son lanzados al mercado mundial.

\section{Conclusiones}

Con la aplicación de la metodología propuesta pudimos construir las figuras $3,4,5$ y 6 que son los medios gráficos que permiten visualizar fácilmente la evolución en la conformación del sistema mundial.

Revisando las figuras mencionadas, podemos concluir que la configuración de la estructura internacional cambia constantemente ya que la misma siempre se ve sujeta a distintas causas y fuerzas externas; impulsadas principalmente por la fuente de energía sistémica que es el motor tecnológico. Para probar esto, es tan solo necesario ver que las tecnologías que dieron origen y sostuvieron a la Revolución Industrial son sustancialmente diferentes de las tecnologías de la información que tuvieron su impacto a principios de la década de los años 90 's del siglo pasado, tales como el internet y la telefonía celular; aún las nuevas innovaciones como la biotecnología, la ingeniería genética, los nuevos materiales y las redes de telecomunicaciones avanzadas, entre muchas otras, son más complejas y diferentes, sin embargo, ellas son las que soportarán y darán vida tanto a la economía como a la sociedad del siglo XXI.

La tecnología nace en las organizaciones humanas, forma parte del quehacer cotidiano de las empresas y de las institu- ciones que realizan investigación y desarrollo. En ellas emergen nuevas tecnologías que con el tiempo pueden modificar las tendencias tanto tecnológicas como de la economía mundial. El quehacer conjunto de individuos, empresas, organizaciones de todo tipo, gobiernos y su relación con otros países en el entorno internacional, define la situación de los diferentes países que conforman el sistema mundial. Al inspeccionar las figuras mencionadas, podemos decir que las ondas- $\mathrm{K}$ son causadas por la demanda de soluciones para nuevos problemas y el suministro de tales soluciones por las empresas innovadoras. El fenómeno se acompasa debido a que los periodos de alto crecimiento de los sectores líderes tienden a provocar la expansión y la prosperidad de la economía general.

Modelski (Op. Cit.), señala que en el año 2013 estamos cursando la tercera década de la onda K-20. Es posible notar que el mercado mundial se está saturando de computadores personales y gadgets por lo que cada vez es más difícil para los productores de estos artefactos ofrecer características innovadoras en sus productos que sean capaces de nuevamente impulsar y dinamizar la economía mundial a gran escala. Si la teoría es correcta, en breve nos encontraremos en la parte de descenso del nuevo ciclo largo económico, por lo que distintos países en el mundo están preparando el terreno para la nueva acometida tecnológica.

En el recuadro de conocimiento de la figura 6 , queda claro que desde finales del siglo pasado y hasta la fecha se han desarrollado intensamente diferentes disciplinas científicotecnológicas; notablemente, uno de los campos del conocimiento que ha tenido una mayor dinámica de desarrollo y que según muchas organizaciones participará en la solución de varios de los problemas actuales tales como el desempleo, la malnutrición y las enfermedades, es la biotecnología. Muchos estudios prospectivos indican que hacia el año 2030 se prevé el crecimiento sostenido de los productos biológicos de alto valor agregado y de las industrias basadas en estos, así como la disponibilidad y la aplicación comercial de los chips biológicos poco a poco dará lugar a la bioconvergencia, tendencia tecnológica que posiblemente provocará el cambio hacia un nuevo sistema económico, la bioeconomía. 


\section{Referencias}

AMERICAN Chemical Society \& The U.S. Chemical Industry, (ACS\&US Chl), (1996). Technology Vision 2020. Department of Government Relations and Science Policy Eds. Washington D. C., pp. 91.

AMIN, S. (2010). Eurocentrism, NYU Press; pp. 240. ISBN: I583673962, 978I583673966.

AMIN, S., Arrighi, G., Frank, A.G., and Wallerstein, (2006) Transforming the Revolution: Social Movements and the World-System, Aakar Books; Pp. 187. ISBN: 8187879947, 9788 I87879947.

BELLO, R. E., (2003). Descartes, R.: Discurso del método; estudio preliminar, traducción y notas. Ed.Tecnos, Madrid.

Berger, P., Luckmann, T., (1966). The social construction of reality: a treatise in the sociology of knowledge, New York; pp. 2 I9. ISBN: | 4532 I5468, 978 |4532 I5463

BESANT,W.A., (I898). La Evolución de la Vida y de la Forma. Disponible en: http://www.logiablavatsky.com/resources/ Annie\%20Besant\%20-\%20La\%20Evolucion.pdf. Consultado 16 junio 2013. First edited as Evolution of life and form: four lectures delivered at the twenty-third anniversary meeting of the Theosophical Society at Adyar, Madras, 1898. Theosophical Pub. Society, London.

COATES, J. F., (2003). Future Innovations in Science and Technology. En "The International Handbook on Innovation”. Larisa V. Shavinina Ed. Elsevier Science Ltd., p.II7I. ISBN: 0-08-044 | 98-X, 978008044| 986.

CORONA, T. L., Jasso,V. J., (2005). Enfoques y características de la sociedad del conocimiento: Evolución y perspectivas para México; en "Innovación en la Sociedad del Conocimiento”. Sánchez, D. G., (Coord.). Benemérita Universidad Autónoma de Puebla y la Red de Investigación y Docencia en Innovación Tecnológica, pp. 34-38.

DRUCKER, P., (2005). Para entender la sociedad del conocimiento. Universidad APEC, Colección UNAPEC, PP. 35. ISBN: 999348I2II, 978999348I2I8.

DUIJN, JJ VAN, (1983). La onda larga en la vida económica, Londres, George Allen and Unwin, Pp. 163.

FARRINGTON, B., (199I). The Philosophy of Francis Bacon: an Essay on Its Development from 1603 to 1609; Liverpool University Press; PP. 139. ISBN: 10-0853233 I0I; 13-9780853233107.
GUNDER A. F, Gills, K. B. (Eds.) (1996). The World System: Five Hundred Years or Five Thousand? Routledge; pp. 320. ISBN: 04I5I50892, 97804I5I50897.

GUNDER A. F, Gills, K. B. (Eds.) (1996). The World System: Five Hundred Years or Five Thousand? Journal of World History.Vol. 7 No. 2. DOI: 10, I 353/jwh.2005.0043

FREEMAN, C., (1985). La revolución de la computadora en los largos ciclos de desarrollo económico, Munich, Carl Friedrich von Siemens Foundation, p. 17.

GOTTFRIED HABERLER (Ed.), ( 1956$)$ Kondrátiev, Nikolái D. 1935: "Los grandes ciclos de la vida económica”; Ensayos sobre el Ciclo Económico: 35-56; Fondo de Cultura Económica, México, $2^{\mathrm{a}} \mathrm{Ed}$.

KERNEY,A.T., (20I2). Chemical IndustryVision 2030:A European Perspective.A.T. Kearney Inc., Korea, Pp. I5. Disponible en: http://www.atkearney.com/documents//0192/536/96/ Chemical+Industry+Vision+2030+A+European+Perspect ive.pdf/7I78bI50-22d9-4b50-9I25-Iflb3a936lef; consultado 30 noviembre 2013

KONDRATIEFF, N. D., (1926). Los ciclos económicos largos, GDP Eds; pp. I I5. ISBN: 0952457 I05, 9780952457 I07.

LAMO DE E. E; González G. J. M; Torres, C., (1994). La sociología del conocimiento y de la ciencia, Alianza Editorial, Madrid, $2^{\text {a }}$ Ed., pp. 636. ISBN 842068|474, 978842068I474.

MARTIN, J., (2006). The meaning of the 2 I st Century: a vital blueprint for ensuring our future. Riverhead Books, Penguin Group, New York, pp. 429. ISBN I-57322-323-9.

MC. LUHAN, M., (200I). Understanding media: The extensions of man. Routledge. New York, Pp.392. ISBN 04I5253977, 97804I5253970

MODELSKI, G. (1987). Long Cycles in World Politics, University of Washington Press; pp. 244.

MODELSKI, G. (|99|). World system evolution: a learning model, paper presented at the 32nd annual meeting of the International Studies Association, Vancouver, 20-23 March.

MODELSKI, G. \& Thompson, W., (2008). Sea Power in Global Politics 1494-1994, London, Macmillan, Pp. 380. ISBN: 0333429257, 9780333429259

MODELSKI, G. \& Thompson, W. R., (1996). Leading Sectors and World Powers: The Coevolution of Global Economics and Politics, Columbia: University of South Carolina Press. USA; PP. 267. ISBN I-57003-054-5.

ISSN: 07I 8-2724. (http://www.jotmi.org) 
NEEDHAM, J., Wang, L., (2004). Science and Civilization in China: Volume 7, The Social Background, Part 2, General Conclusions and Reflections. Cambridge University Press, Pp. 283. ISBN: 052 I087325, 978052 I 087322.

NEFIODOW, L. A., (2006). Der sechste Kondratieff. Wege zur Produktivität und Vollbeschäftigung im Zeitalter der Information. Sixth Edition, Rhein-Sieg-Vlg Nefiodow Eds; pp. 303. ISBN: 39805 I 4455, 97839805 I 4453.

NEFIODOW, L. A., (20I3). The Sixth Kondratieff. Disponible en http://www.kondratieff.net/l I.html, Consultado 07 julio20I3.

SCHUMPETER, J.A. (20I2). The theory of economic development: an inquiry into profits, capital, credit, interest, and the business cycle, Sixteehnth Edition. Transactions Publishers Rutgers, New Jersey USA; Pp. 255, ISBN 978-0-87855698-4

TAIT, J., WIELD, D., Bruce A., Chataway, J., (2009). Health Biotechnology to 2030. OECD International Futures Project on "The Bioeconomy to 2030: Designing a Policy Agenda". OECD Publishing, Pp.323. ISBN: 926403853I, 9789264038530.

WALLERSTEIN, I. (1984). The Politics of the World-Economy, Cambridge University Press. USA; Pp. 191. ISBN: 052I277604, 9780521277600.

WALLERSTEIN, I. (20I I). The Modern World-System, Vol. I, University of California Press. USA; PP. 4I0. ISBN: 978-0520-26757-2.

WALLERSTEIN, I. (20II). The modern world System III: The second era of great expansion of the capitalist world economy 1730's- 1840's. University of California Press; pp. 390. ISBN: $0520948599,9780520948594$. 